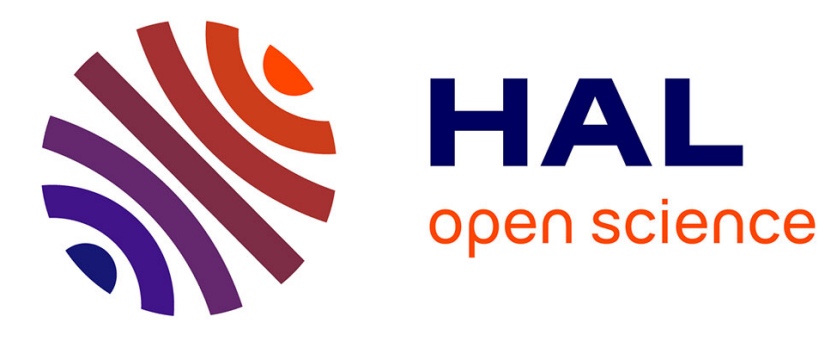

\title{
Multiple collisions in turbulent flows
}

Michel Vosskuhle, Emmanuel Lévèque, Michael Wilkinson, Alain Pumir

\section{To cite this version:}

Michel Vosskuhle, Emmanuel Lévèque, Michael Wilkinson, Alain Pumir. Multiple collisions in turbulent flows. Physical Review E: Statistical, Nonlinear, and Soft Matter Physics, 2013, 88, pp.063008. 10.1103/PhysRevE.88.063008 . hal-00931521

\section{HAL Id: hal-00931521 \\ https://hal.science/hal-00931521}

Submitted on 11 Apr 2016

HAL is a multi-disciplinary open access archive for the deposit and dissemination of scientific research documents, whether they are published or not. The documents may come from teaching and research institutions in France or abroad, or from public or private research centers.
L'archive ouverte pluridisciplinaire HAL, est destinée au dépôt et à la diffusion de documents scientifiques de niveau recherche, publiés ou non, émanant des établissements d'enseignement et de recherche français ou étrangers, des laboratoires publics ou privés. 


\title{
Multiple collisions in turbulent flows
}

\author{
Michel Voßkuhle, ${ }^{1}$ Emmanuel Lévêque, ${ }^{1,2}$ Michael Wilkinson, ${ }^{3}$ and Alain Pumir ${ }^{1}$ \\ ${ }^{1}$ Laboratoire de Physique, ENS de Lyon and CNRS, UMR5672, 46, allée d'Italie, F-69007 Lyon, France \\ ${ }^{2}$ Laboratoire de Mécanique des Fluides et d'Acoustique, Ecole Centrale de Lyon and CNRS, UMR5509, \\ 36 avenue Guy de Collonge F-69134, Ecully, France \\ ${ }^{3}$ Department of Mathematics and Statistics, The Open University, Walton Hall, Milton Keynes, MK6 7AA, England
}

(Received 29 September 2013; published 10 December 2013)

\begin{abstract}
In turbulent suspensions, collision rates determine how rapidly particles coalesce or react with each other. To determine the collision rate, many numerical studies rely on the ghost collision approximation (GCA), which simply records how often pairs of point particles come within a threshold distance. In many applications, the suspended particles stick (or in the case of liquid droplets, coalesce) upon collision, and it is the frequency of first contact which is of interest. If a pair of "ghost" particles undergoes multiple collisions, the GCA may overestimate the true collision rate. Here, using fully resolved direct numerical simulations of turbulent flows at moderate Reynolds number $\left(\operatorname{Re}_{\lambda}=130\right)$, we investigate the prevalence and properties of multiple collisions. We find the probability $P\left(N_{c}\right)$ for a given pair of ghost particles to collide $N_{c}$ times to be of the form $P\left(N_{c}\right)=\beta \alpha^{N_{c}}$ for $N_{c}>1$, where $\alpha$ and $\beta$ are coefficients which depend upon the particle inertia. We also investigate the statistics of the times that ghost particles remain in contact. We show that the probability density function of the contact time is different for the first collision. The difference is explained by the effect of caustics in the phase space of the suspended particles. We demonstrate that, as a result of multiple collisions, the GCA leads to a small, but systematic overestimate of the collision rate, which is of the order of $\sim 15 \%$ when the particle inertia is small, and slowly decreases when inertia increases.
\end{abstract}

DOI: 10.1103/PhysRevE.88.063008

PACS number(s): 47.27.-i, 05.40.-a, 45.50.Tn, 92.60.Mt

\section{INTRODUCTION}

Collisions between particles transported by a turbulent flow play a crucial role in several important phenomena. Saffman and Turner [1] suggested that turbulence in clouds can lead to a very significant enhancement of the rate of collision between small droplets. This mechanism has been proposed to provide an explanation for the very fast rate of coalescence reported in warm cumulus clouds [2-4]. Also, collisions between dust grains in a turbulent circumstellar accretion disc play an important role in some theories for planet formation $[5,6]$.

There is a substantial literature on the collision rate of particles in turbulent flows. Most of the studies devoted to collisions in turbulent suspensions explicitly deal with "geometric collisions," that is, merely detect when the centers of two particles are separated by a distance $d$ less than the sum of their radii, $a_{1}+a_{2}$. The time-dependent separation $d(t)$ may cross $a_{1}+a_{2}$ repeatedly, and every crossing from above is considered as a new collision. In many applications, however, the colliding particles are assumed to stick, coalesce, or react on first contact. In these cases the multiple collisions are spurious, and the physically relevant collision rate should count only the frequency with which $d(t)$ decreases below $a_{1}+a_{2}$ for the first time. The "ghost collision approximation" (GCA) consists in ignoring this aspect and in following all particles in the flow even after they underwent collisions. While this approximation is appealing from a numerical point of view, it has been noticed to lead to questionable estimates of the collision rate $[7,8]$, because it includes spurious multiple collisions [9].

The first objective of our study is to characterize the statistics of the multiple collisions. This question is particularly relevant when studying model classes of reaction occurring with a finite probability when particles are close enough
[10]. We therefore investigate the statistics of the number of contacts, $N_{c}$. We find that there is a very simple distribution of the number of times a pair of particles collides: the probability for observing $N_{c}$ collisions after one initial collision is found to be well approximated by

$$
P\left(N_{c} \mid N_{c} \geqslant 1\right)=\beta \alpha^{N_{c}},
$$

where the coefficients $\alpha$ and $\beta$ depend upon parameters as discussed below.

It has been observed that tracer particles in a turbulent flow can remain in proximity for a long time [11-13]. It is natural to expect that this phenomenon may be related to the multiple collisions which we are investigating here. With this motivation, we consider the statistics of the time that a pair of ghost particles is in contact and relate this to the distribution of relative velocities of collisions. The probability distribution function (PDF) of the contact time exhibits a striking structure. For the first collision, it follows a power law at intermediate values and an exponential decay at longer contact times. The PDFs of the contact time for the first collision are markedly different from those of subsequent collisions (which lack the power-law behavior and which appear to be independent of the number $N_{c}$ of collisions, for $N_{c} \geqslant 2$ ). We explain this discrepancy by appealing to a model for the collision process introduced in Refs. [2,4], according to which the collision rate $\Gamma$ can be expressed as the sum of two terms, resulting from two different types of physical processes:

$$
\Gamma=\Gamma_{\text {adv }}+\Gamma_{\text {caust }} .
$$

The term $\Gamma_{\text {adv }}$ represents the rate of collisions of particles which are advected into contact by shearing motion due to turbulence. This advective process allows for the possibility of multiple collisions, because the local velocity gradient 
can fluctuate as a function of time, so that particles may be brought back into contact after separating for a while. The term $\Gamma_{\text {caust }}$ results from the possibility that particles with significant inertia can move relative to the fluid. If caustics (fold lines) form in the phase space of the suspended particles, particles occupying the same position may have different velocities. This phenomenon can also be understood as resulting from particles being centrifuged out of vortices and has also been referred to as the "sling" effect [2,14]. Experiments have recently demonstrated the existence of the "sling" effect in a well-controlled laboratory flow [15]. Particles colliding at higher relative velocity are unlikely to undergo multiple collisions. The higher-velocity collisions generated by caustics contribute to the statistics of the first collision, but not to the multiple collisions. The use of the decomposition (2) is supported by work demonstrating that it is valid for a random flow model [16,17]. A companion to this paper [18] shows that (2) is a good model for DNS studies of turbulence and discusses the relative importance of the two terms as a function of the parameters of the turbulent suspension.

What happens to two particles after they have come into contact depends on the precise physical context [10,19]. We are interested here in problems where particles have a strong probability of reacting after their first contact. In these cases the multiple encounters between a given pair of particles, quantified by $\Gamma_{m}$, correspond to an unphysical contribution to the collision rate. Let $\Gamma_{1}$ be the rate for first contact collisions, and $\Gamma_{m}$ be the rate for subsequent multiple collisions. The GCA collision rate can be written as a sum:

$$
\Gamma_{\mathrm{GCA}}=\Gamma_{1}+\Gamma_{m} .
$$

The existence of multiple collisions points to a shortcoming of the GCA: when particles coalesce upon collision, $\Gamma_{1}$ is the physically relevant collision rate, and the rate of multiple collisions $\Gamma_{m}$ is a spurious contribution to $\Gamma_{\mathrm{GCA}}$.

One way to evaluate the true collision rate $\Gamma_{1}$ is to count the rate of multiple collisions and subtract it from $\Gamma_{\mathrm{GCA}}$. We have also considered two other processes, which lead to alternative definitions. The first process consists in assuming explicitly that the two particles do not participate any longer to the reactions as soon as they have come into contact, as if they had annihilated. Technically, in order to deal with a steady-state system, we introduce two new particles to compensate for the loss. The implementation of this algorithm requires some care in the case of inertial particles, as the velocity depends on history. Another process consists in removing and replacing only one of the two colliding particles. We demonstrate here that the collision rates describing these two processes are equal to $\Gamma_{1}$, in the limit of very dilute suspensions, which justifies its relevance.

The results presented here very significantly extend a previous study, using a simpler model of turbulent flow, namely the kinematic simulation approach [20]. While the results concerning the relative errors made by using this simplified flow are qualitatively similar to the results presented in this work, we find that kinematic simulations lead to a very significant underestimation of the collision rates, by almost an order of magnitude.

Our work emphasizes models where the suspended particles coalesce upon contact, so that multiple collisions are a source of error in the collision rate. These models are just a limiting case of a larger class of models, which may also be of physical interest. As an example, the case of fully elastic collisions was recently considered in Ref. [19], which showed that elastic collisions lead to a finite probability of particles making an infinite number of collisions. Our investigations of the contact times in the GCA involve evaluating the distribution of the time that one particle in a turbulent flow spends within a sphere surrounding another particle. We remark that Jørgensen et al. [21] have investigated this distribution experimentally in a different context, where the radius of the sphere is much larger than the Kolmogorov length of the flow. Statistics of multiple collisions have been investigated in a different context [22].

This article is organized as follows. In Sec. II we present the numerical schemes used to simulate the carrying turbulent flow and account for the dynamics and collisions of the suspended particles. We describe the algorithms used to study the physical problem of particles coagulating during their first contact and establish that the reaction rate reduces, in the very limit, to $\Gamma_{1}$. The nature of the correction due to a finite particle density is discussed in depth in the Appendix. The quantitative estimates of the probability for a given pair of particles to undergo multiple collisions are discussed in Sec. III, together with the data justifying the empirical law (1). These results lead to estimates of the error made by using the GCA. Section IV is devoted to the statistics of the multiple collisions. Section V discusses the explanation for some of the observations in terms of statistics of the relative velocities upon collision. Finally, we summarize our results in Sec. VI.

\section{NUMERICAL METHODS}

\section{A. Direct numerical simulation of Navier-Stokes turbulence}

The work rests on simulating the (incompressible) NavierStokes equations:

$$
\begin{gathered}
\partial_{t} \boldsymbol{u}(\boldsymbol{x}, t)+(\boldsymbol{u}(\boldsymbol{x}, t) \cdot \nabla) \boldsymbol{u}(\boldsymbol{x}, t) \\
=-\nabla p(\boldsymbol{x}, t)+v \nabla^{2} \boldsymbol{u}(\boldsymbol{x}, t)+\boldsymbol{f}(\boldsymbol{x}, t), \\
\boldsymbol{\nabla} \cdot \boldsymbol{u}(\boldsymbol{x}, t)=0,
\end{gathered}
$$

where $\boldsymbol{u}(\boldsymbol{x}, t)$ denotes the Eulerian velocity field, $v$ is the viscosity, and $\boldsymbol{f}(\boldsymbol{x}, t)$ is a forcing term; the mass density is arbitrarily set to unity. These equations are solved in a cubic box of size $2 \pi$ with periodic boundary conditions in the three directions by a pseudospectral method. The pressure $p(\boldsymbol{x}, t)$ is eliminated by taking the divergence of (4) and by solving the resulting Poisson equation in the spectral domain [23]. The forcing term acts on Fourier modes of low wave numbers, $|\boldsymbol{k}| \leqslant K_{f}$. It is adjusted in such a way that the injection rate of energy, $\varepsilon$, remains constant [24]:

$$
\boldsymbol{f}_{\boldsymbol{k}}=\varepsilon \frac{\boldsymbol{u}_{\boldsymbol{k}}}{\sum_{|\boldsymbol{k}| \leqslant K_{f}}\left|\boldsymbol{u}_{\boldsymbol{k}}\right|^{2}} \quad \text { if } \quad|\boldsymbol{k}| \leqslant K_{f} .
$$

The simulations discussed in this study have been done with the following parameters, in code units: $\varepsilon=10^{-3}$ and $v=4 \times 10^{-4}$. The forcing operates at wavenumbers $|\boldsymbol{k}| \leqslant$ $K_{f}=1.5$. With these values, the Kolmogorov scale, $\eta_{\mathrm{K}}=$ $\left(v^{3} / \varepsilon\right)^{1 / 4}$, is comparable to the effective spatial resolution $\Delta x=2 \pi / 256: \Delta x / \eta_{\mathrm{K}} \approx 1.5$ or $k_{\max } \eta \approx 2.1$, which fulfills 
standard requirements for the direct numerical simulation (DNS) of Navier-Stokes turbulence and the integration of particle trajectories [25]. Let us notice that, in practice, the number of grid points is 384 in each direction according to the two-thirds rule [26] (used to avoid aliasing errors). However, padded high-wavenumber modes are not excited and, therefore, do not contribute to improving the spatial resolution of the solution. With these values of the parameters, the value of the Reynolds number based on the Taylor microscale is $\operatorname{Re}_{\lambda} \approx 130$ in the statistically stationary longtime limit state.

After spectral truncation, Eq. (4) reduces to a set of ordinary differential equations (in time) for the Fourier modes, which have been integrated using the second-order Adams-Bashforth scheme. The time step $\delta t$ has been chosen so that the Courant number $\mathrm{Co}=u_{\mathrm{rms}} k_{\max } \delta t \lesssim 0.1$. With this choice, the relaxation time $\tau_{p}$ of the particle dynamics remains of the order of $10^{2} \delta t$, which ensures that particle trajectories can be safely integrated from the time-evolving Eulerian velocity field.

\section{B. Dynamics of particles}

We simulated in our flow the motion of small particles, all of which are assumed to have the same size and mass, whose motion obeys the following set of equations:

$$
\frac{d \boldsymbol{x}}{d t}=\boldsymbol{v}, \quad \frac{d \boldsymbol{v}}{d t}=\frac{\boldsymbol{u}(\boldsymbol{x}, t)-\boldsymbol{v}}{\tau_{p}} .
$$

This set of equations is a simplified version of the original set derived by Refs. [27,28] and is appropriate for small spherical particles with radius $a$, which is much smaller than the Kolmogorov scale $\eta_{\mathrm{K}}$, and whose density is much larger than the fluid density: $\rho_{p} / \rho_{f} \gg 1$. In order to isolate the role of turbulence, we have explicitly neglected gravity in Eq. (7), despite the fact that it plays an important role in cloud microphysics, sandstorms, and other terrestrial phenomena. Gravitational effects on collision rates are unimportant in applications to planet formation. The relaxation time in Eq. (7) is determined by the Stokes drag:

$$
\tau_{p}=\frac{2}{9} \frac{\rho_{p}}{\rho_{f}} \frac{a^{2}}{v} .
$$

This relaxation time is made dimensionless by using the Kolmogorov time scale, $\tau_{\mathrm{K}}=(v / \varepsilon)^{1 / 2}$, and the Stokes number

$$
\mathrm{St}=\frac{\tau_{p}}{\tau_{\mathrm{K}}}
$$

is a dimensionless measure of the importance of inertial effects in determining the trajectories of the particles. In order to explore parameter ranges which are relevant to cloud microphysics, we used a ratio of densities $\rho_{p} / \rho_{f}=10^{3}$ throughout. Lengths and times are given in dimensionless units and can be readily scaled to realistic situations.

The determination of the particle velocity $\boldsymbol{v}$ requires, according to (7), the evaluation of the fluid velocity $\boldsymbol{u}$ at the location of the particle. This is done by resorting to tricubic interpolation. The particle trajectories have been integrated by using the second-order Verlet velocity algorithm [29].

As we are interested in determining the collision rates in turbulent flows, we simulated a large number of particles. The number of particles $N_{p}$ used to monitor the collision rates was chosen in such a way that the volume fraction $\Phi=$ $4 N_{p} \pi a^{3} /\left(3 L^{3}\right)$, where $L$ is the size of the system $(L=2 \pi)$, is either $\Phi=4.5 \times 10^{-6}$ or $\Phi=4.5 \times 10^{-5}$. In both cases, collisions involving three or more particles can be neglected. Note that the particle radius $a$ is entirely specified by the Stokes number, via (8) and (9), and the number of particles $N_{p}$ is fixed by the definition of $\Phi$, once $a$ is known.

In a flow having reached a statistically steady state, we inserted at a time $T=0$ a total number $N_{p}^{\prime} \simeq 1.1 N_{p}$ of particles, initially distributed uniformly in the flow. The reason why we simulate more particles than we actually use for monitoring collisions will be explained in Sec. II C.

We then integrated the equations of motion (4) and (7), for a time of the order of 10 eddy-turnover times, $T_{L}$, defined by

$$
T_{L}=\frac{L}{\sqrt{\left\langle\boldsymbol{u}^{2} / 3\right\rangle}}, \quad \text { with } \quad L=\frac{3 \pi}{2\left\langle\boldsymbol{u}^{2}\right\rangle} \int k^{-1} E(k) d k
$$

After this time, we integrated the equations of motion for a time $t_{\text {tot }}$ larger than $15 T_{L}$ (except for $\mathrm{St}=0.2$ ). All the particle trajectories were saved, with a sampling time of $\Delta t \approx 0.055 \tau_{\mathrm{K}}$, and processed afterward. Table I summarizes our runs.

For the case of $\mathrm{St}=0$, we integrated the trajectories of Lagrangian tracer particles. Those are point particles with no extent, but to determine the collision rate it is necessary to assume they have a finite size. We chose the radius to be the same as for particles with $\mathrm{St}=0.1$.

\section{Detection of the collisions}

In a monodisperse solution of volume $V$, containing $N_{p}$ particles, the average number of collisions $\mathcal{N}_{c}$ per unit of time and per unit volume is proportional to the square of the average number of particles per unit volume, $n \equiv N_{p} / V$ :

$$
\mathcal{N}_{c}=\Gamma_{c} \frac{n^{2}}{2} .
$$

Equation (11) defines the collision kernel $\Gamma_{c}$, which depends both on the fluid motion, and on the physical properties of the

\begin{tabular}{|c|c|c|c|c|c|c|c|c|c|c|c|c|c|c|}
\hline St & 0.0 & 0.10 & 0.20 & 0.30 & 0.51 & 0.76 & 1.01 & 1.27 & 1.52 & 2.03 & 2.53 & 3.04 & 4.05 & 5.07 \\
\hline$\Phi \times 10^{6}$ & 4.5 & 4.5 & 45 & 4.5 & 45 & 45 & 45 & 45 & 45 & 45 & 45 & 45 & 45 & 45 \\
\hline$t_{\mathrm{tot}} / T_{L}$ & 15.5 & 15.5 & 4.2 & 31.4 & 15.9 & 10.4 & 47.6 & 13.0 & 52.4 & 52.1 & 52.5 & 52.3 & 53.1 & 42.1 \\
\hline$N_{\mathrm{GCA}} / 10^{4}$ & 0.6 & 1.3 & 29 & 3.3 & 200 & 180 & 400 & 81 & 250 & 150 & 99 & 72 & 42 & 22 \\
\hline
\end{tabular}
particles.

TABLE I. Summary of parameters from our different DNS runs. We tabulate the Stokes number St, the volume fraction occupied by the particles $\Phi$, the total integration time $t_{\text {tot }}$ used to determine the collision rate, expressed in terms of the large eddy turnover time $T_{L}$, and the total number of collisions $N_{\mathrm{GCA}}$ detected, when using the GCA. 
The particle trajectories determined numerically were stored and postprocessed separately, to determine the number of collisions and other statistics. Detecting collisions requires checking the mutual distance between $N_{p}$ particles, which typically requires of the order of $N_{p}^{2}$ operations. In simulations such as ours involving a large number of particles, this can lead to a prohibitively large computational time. We used an algorithm based on a cell-linked list to speed up the processing time, as done in Ref. [30].

Each data set was processed in two different ways:

(1) Multiple collisions of ghost particles. First, we determined the collision rate using the GCA. In this case only $N_{p}$ of the total $N_{p}^{\prime}$ simulated particles were used. The collision kernel $\Gamma_{\mathrm{GCA}}$ was simply determined by counting the total number of detected collisions $\mathbf{N}_{c}$. In addition, we also determined the rates at which pairs of particles come into contact for the $N_{c}$ th time, described by the collision kernel $\Gamma_{N_{c}}$. To this end, at each collision between two particles, we examine the trajectories leading up to the collision and determine the number of previous collisions between the same pair. If the pair has undergone $N_{c}-1$ previous collisions with each other, the collision event contributes to the kernel $\Gamma_{N_{c}}$. Because these collision kernels describe an exhaustive and mutually exclusive decomposition of $\Gamma_{\mathrm{GCA}}$, we have

$$
\Gamma_{\mathrm{GCA}}=\sum_{N_{c}=1}^{\infty} \Gamma_{N_{c}} .
$$

The collision kernel of multiple collisions $\Gamma_{m}$ is simply defined by summing the kernels $\Gamma_{N_{c}}$, for $N_{c} \geqslant 2$ :

$$
\Gamma_{m}=\sum_{N_{c}=2}^{\infty} \Gamma_{N_{c}} .
$$

(2) Collision detection with particle replacement. We have assumed that, in the case of particles coalescing or reacting on their first contact, $\Gamma_{1}$ is the correct measure of the collision rate. To establish this result, we compared $\Gamma_{1}$ with a collision rate algorithm where particles are removed from the flow after collision. We determined the collision rate again with $N_{p}$ particles, but we systematically replaced either one or two of the colliding particles after each collision. The substitutions are carried out by simply picking one of the $\left(N_{p}^{\prime}-N_{p}\right)$ noncolliding particles, making sure that the newly introduced particle is not colliding with any other particle at the moment of its insertion. In so doing, the collision rate is determined at a fixed density. A similar method has been used in Refs. [7,31]. The rates determined by this procedure are denoted $\Gamma_{\mathrm{Re} 1}$ and $\Gamma_{\operatorname{Re} 2}$, depending upon whether one or two particles are replaced. We stress that the simplest method, consisting in generating new particles at random positions, cannot work in the case of finite inertia $(\mathrm{St} \neq 0)$, as the velocity depends on history. Simulating $N_{p}^{\prime}>N_{p}$ particles allows us to deal only with particles which are already in equilibrium with the flow. We refer in the following to these algorithms as the one- or two-particle "substitution schemes."

It is not immediately clear that $\Gamma_{\operatorname{Re} 1}=\Gamma_{\operatorname{Re} 2}=\Gamma_{1}$. In fact, our numerical results reveal measurable deviations from these identities at the highest particle volume fraction studied here. However, as we explain in detail in the Appendix, these deviations decrease linearly when reducing the particle density, thus establishing that $\Gamma_{1}=\Gamma_{\mathrm{Re} i}$ in the limit of very dilute suspensions.

In all cases the number of collisions $\mathbf{N}_{c}(\Delta \tau)$ is measured in consecutive intervals of length $\Delta \tau \sim T_{L}$. For each interval the collision kernel can be determined as

$$
\langle\Gamma\rangle_{\Delta \tau}=\frac{2 V \mathbf{N}_{c}(\Delta \tau)}{\Delta \tau N_{p}^{2}},
$$

which is simply the collision rate divided by $n^{2} / 2$. These "instantaneous" collision kernels vary in time. Determining the level of fluctuations of the number of collisions recorded over a limited time interval led to an estimate of the uncertainty of the collision rate. The resulting uncertainty in the numerical value of $\Gamma_{\mathrm{GCA}}$ is less than $2 \%$, except for $\mathrm{St}=0.2$ and $\mathrm{St}=0.5$, where it is no larger than $4 \%$.

\section{QUANTIFYING MULTIPLE COLLISIONS BETWEEN A GIVEN PARTICLE PAIR}

\section{A. Relative contribution of multiple collisions and error of the GCA}

In this section we discuss the probability of having multiple collision between a given pair of particles and relate this to the error induced by using the GCA. The left panel of Fig. 1 shows the collision rate computed by using the GCA (curve with square symbols) and the collision rate $\Gamma_{1}$ (curve with circle symbols). Our estimates of the collision rates compare quantitatively very well with previous numerical work [32,33]. The right panel shows the relative difference $\Gamma_{m} / \Gamma_{1}=$ $\left(\Gamma_{\mathrm{GCA}}-\Gamma_{1}\right) / \Gamma_{1}$.

Our results show that among all the collisions recorded, as many as $15 \%$ of them involve pairs of particles that have already come into contact. We note that our results show that the collision rate determined by using the GCA is correctly approximated by the Saffman-Turner formula when $\mathrm{St} \rightarrow 0$, consistent with previous results [7]. However, in the limit of small inertia, the property that pairs of particles collide more than one time is very significant. This probability decreases when the Stokes number increases. This result can be qualitatively understood by using the known fact that when the Stokes number increases, the particle trajectories differ more from the fluid trajectories, which allows collisions between particles with an increasingly large velocity difference. Colliding particles are therefore expected to separate quicker when the Stokes number is large, thus making multiple collisions less likely.

In physical situations where particle pairs react upon first contact, the collision rate $\Gamma_{1}$ is expected to provide the correct estimate of the reaction rate. To this end, we have measured the rates $\Gamma_{\mathrm{Re}_{1}}$ and $\Gamma_{\mathrm{Re}_{2}}$, defined by taking one or two of the colliding particles out of the system after collisions. The values of $\Gamma_{\mathrm{Re}_{1}}$ and $\Gamma_{\mathrm{Re}_{2}}$ are found to be actually smaller than $\Gamma_{1}$. In fact, as we explain in the Appendix, the difference between $\Gamma_{\mathrm{Re}_{i}}(i=1,2)$ and $\Gamma_{1}$ is due to close encounters between three particles, an effect whose relative importance becomes weaker when the total density of particles goes to zero. The analysis presented in the Appendix establishes that in the limit of a very dilute system, the proper collision rate is indeed $\Gamma_{1}$. 

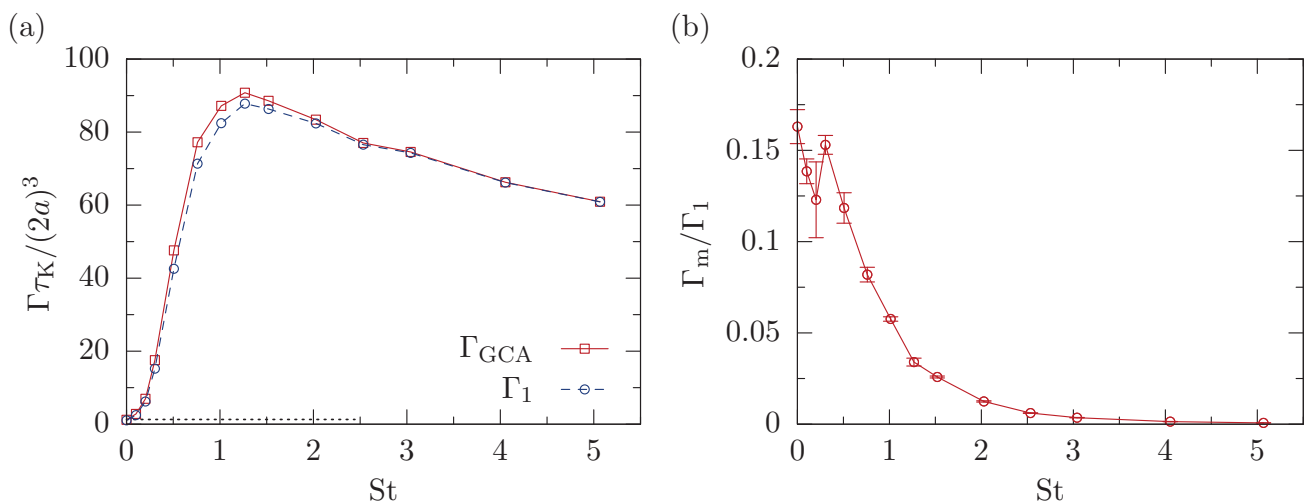

FIG. 1. (Color online) Comparison between the collision kernels $\Gamma_{\mathrm{GCA}}$ and $\Gamma_{1}$. The collision kernel $\Gamma_{\mathrm{GCA}}$ in panel (a) was obtained according to the GCA, taking into account all collisions. The collision kernel $\Gamma_{1}$ is restricted to only first collisions of a same pair. The difference, $\Gamma_{m}=\Gamma_{\mathrm{GCA}}-\Gamma_{1}$, which corresponds to the relative error introduced by the GCA, is shown in the left panel. This error starts at a value close to $\sim 15 \%$ when $\mathrm{St} \rightarrow 0$ and decreases for higher Stokes numbers.

\section{B. Statistics of multiple collision}

In view of the importance of multiple collisions between a pair of particles, we characterize here the statistical properties of the number of collisions a given pair of particles undergoes before separating. Figure 2 shows the probability distribution function for a particle pair to collide an $N_{c}$ th time after at least one initial collision. The clear result from Fig. 2 is that for $N_{c} \geqslant 2$, the probability distribution is very well approximated by an exponential law of the form of Eq. (1). This remarkably simple functional form leads to the interpretation that once a pair of particles has undergone more than one collision, it has a probability $\alpha$ to collide once more before it separates, the quantity $\alpha$ being independent of $N_{c}$. This suggests a Markovian process of multiple collisions, amenable to a simple modeling.

The exponential law, Eq. (1), can be used to sum the series in Eq. (12) and hence to determine the error of the $\Gamma_{\mathrm{GCA}}$ estimate. We have $\Gamma_{N_{c}}=\Gamma_{1} \beta \alpha^{N_{c}}$ for $N_{c}>1$, so that

$$
\Gamma_{m}=\Gamma_{1} \beta \sum_{N_{c}=2}^{\infty} \alpha^{N_{c}}=\Gamma_{1} \frac{\alpha^{2} \beta}{1-\alpha} .
$$

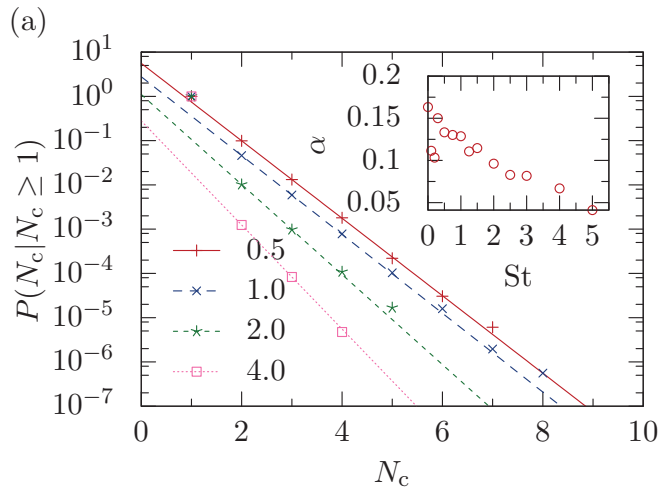

The numerical values obtained from expression (15), with the values of $\alpha$ and $\beta$ extracted from the probability of $N_{c}$ [see Fig. 2(a)], agree quantitatively very well with the value of $\Gamma_{m}$ determined directly [see Fig. 2(b)]. The error bars of $\sim 5 \%$ in the determination of $\Gamma_{m}$, shown in Fig. 1(b), and of the values of $\alpha$ and $\beta$, can easily explain the difference of less than $\sim 10 \%$ between the two curves in Fig. 2(b).

\section{STATISTICS OF THE CONTACT TIME BETWEEN PARTICLE PAIRS}

Our numerical observation that a given pair of particles may collide many times in a turbulent flow can be related to some surprising properties of particle trajectories, which have been partly documented before [11-13] mostly in the case of tracers $(\mathrm{St}=0)$. In this section we fully characterize several properties concerning the time particle pairs spend together, which is relevant to the subject of the present article.

\section{A. Particle trajectories can stay close together for a long time}

Figure 3 shows the distance between two pairs of particles over a long time. Panel (a) shows the distance in units of

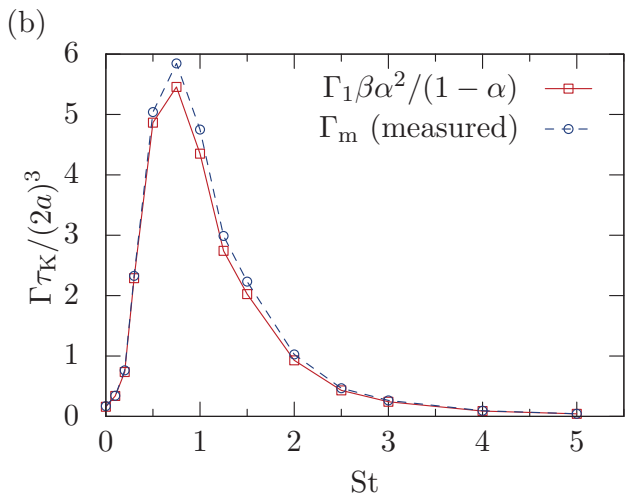

FIG. 2. (Color online) (a) The probability that a pair of particles collides an $N_{c}$ th time conditioned on the fact that it collides at least once. The probability of observing $N_{c}$ collisions goes as $\beta \alpha(\mathrm{St})^{N_{c}}$. The results are shown at different Stokes numbers. The values of $\alpha(\mathrm{St})$ are shown in the inset of the figure as a function of St. (b) The kernel for multiple collisions $\Gamma_{m}$ determined from Eq. (15) with the fitting parameters deduced from panel (a) (square symbols) and measured directly in our simulations (circles). The quantitative agreement between both results confirms the consistency of our reasoning and the quality of our fits. 
(a)

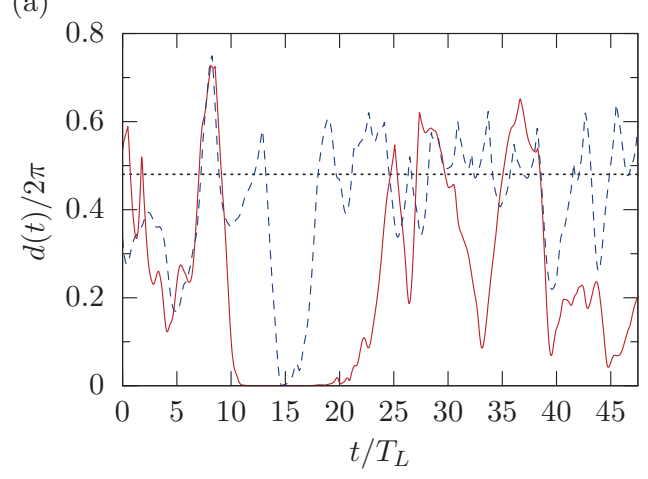

(b)

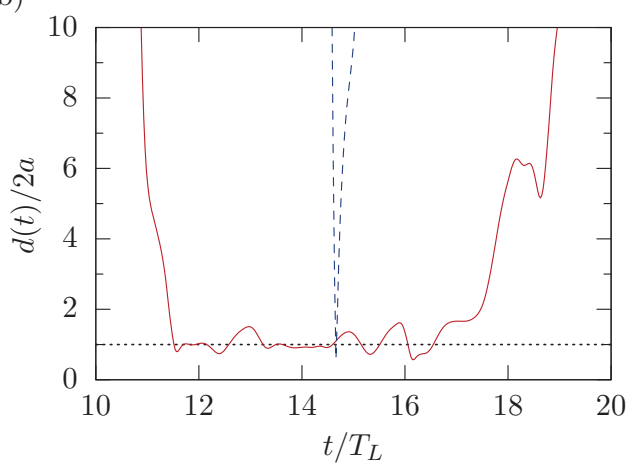

FIG. 3. (Color online) Distance between two different pairs of particles as a function of time. (a) The distance over several eddy turnover times, showing that particles can be close to each other for a short time (dashed curve) or for a much longer time (full line), corresponding to several large eddy turnover times $T_{L}$. (b) Magnifying the graph shown in (a) in the region where the distance between the particles is very small. The solid line reveals that multiple collisions occur. The dotted line in (a) is the average distance for particle pairs homogeneously distributed in a periodic cube. In (b) the dotted line indicates the collision radius $2 a$.

the size of the periodic box during one entire simulation. As expected, the particles are far from each other for most of the time. However, we notice that the distance can reach a very small value at some particular moments. Whereas one of the pairs of particles (dashed line) is close to each other only for a short while, the other pair spends a much longer time close to each other. Panel (b) blows up the result in the range of time where the distance is small. (Here the distance is shown in terms of the collision radius $2 a$.) The pair whose distance is plotted with a dashed line reaches the value of $2 a$, i.e., collides, but bounces apart immediately. In comparison, the continuous curve shows that the two particles stay together for a time larger than the large eddy-turnover time. Over this period of time, the distance fluctuates close to the value of $2 a$, which causes multiple collisions between the two particles. The value of the Stokes number of the particles shown in Fig. 3 is $\mathrm{St}=1.0$; the phenomenon shown here is qualitatively similar at different Stokes numbers.

We observe that the time scale for these multiple collision processes is comparable to the turnover time of the largest eddies in our simulation $T_{L}$. In the following investigations, we have expressed our results in terms of a dimensionless time $t / T_{L}$. However the ratio between $T_{L}$ and the Kolmogorov time scale, $\tau_{\mathrm{K}}$, in our simulations is not very large: $T_{L} / \tau_{\mathrm{K}} \approx$ 10.5. At present it is unclear whether $t / T_{L}$ is the most natural dimensionless time scale. We remark that a plausible alternative is $t \lambda_{1}$, where $\lambda_{1}$ is the leading Lyapunov exponent. Because $\lambda_{1} \approx 0.15 / \tau_{\mathrm{K}}$ [34], the variables $t / T_{L}$ and $t \lambda_{1}$ are quite similar in magnitude.

\section{B. Distribution of contact times}

The phenomenon reported here is very reminiscent of the observation discussed in several earlier works [11-13], namely, that particles can stay close for a very long time. While this property has been documented mostly in the case of tracers, our observations suggest that inertial particles can also remain very close for a long time. As has been noticed in a slightly different context [10], it is of general interest to characterize the statistical properties of the time that particles spend together.
In this subsection we determine quantitatively the statistical properties of the time particle trajectories remain close to one another. We illustrate in the two following subsections the statistics at a fixed value $\mathrm{St}=1.5$ of the Stokes number.

Figure 4 introduces our notation. We consider a pair of particles which become closer than a threshold distance $d_{c}$ at an instant of time. We denote by $t_{e, 1}$ the first instant for which the distance between the particles becomes less than $d_{c}$, (that is, the instant of their first collision) and then $t_{s, 1}$ the first instant $t_{s, 1}>t_{e, 1}$ when they separate. If particles approach to within a distance $d_{c}$ again at some later time, we denote by $t_{e, 2}$ the first instant for which the distance becomes again smaller than $d_{c}$, and $t_{s, 2}$ the time at which the particles separate again. This notation can be easily generalized when the trajectories become more than two times closer than $d_{c}$. The time particles spend together during their first encounter is denoted $\Delta T_{1} \equiv\left(t_{s, 1}-t_{e, 1}\right)$, which again, can be easily generalized to the time particles spend together during their $n$th encounter.

Figure 5 shows the distribution of the time particles spend together during their first encounter $\Delta T_{1}$. The data correspond to particles with a Stokes number $\mathrm{St}=1.5$. Panel (a) shows the PDF of $\Delta T_{1}$ in lin-log scale. In this figure, $\Delta T_{1}$ is expressed

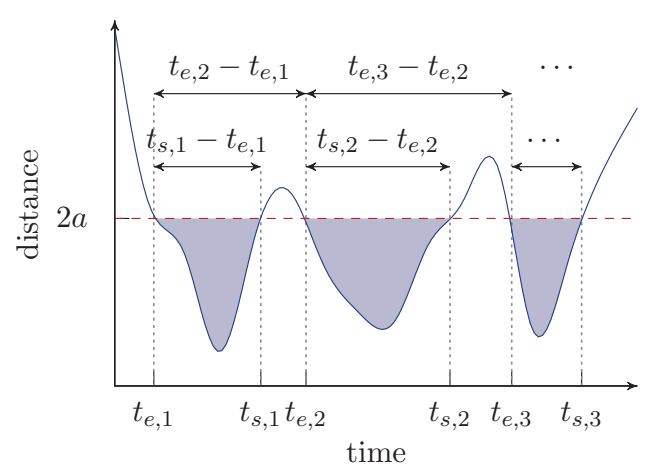

FIG. 4. (Color online) Illustration of the definition of the different times: time of first encounter $t_{e, 1}$, time of first separation $t_{s, 1}$, time of second encounter $t_{e, 2}$, and time of second separation $t_{s, 2}$. 
(a)

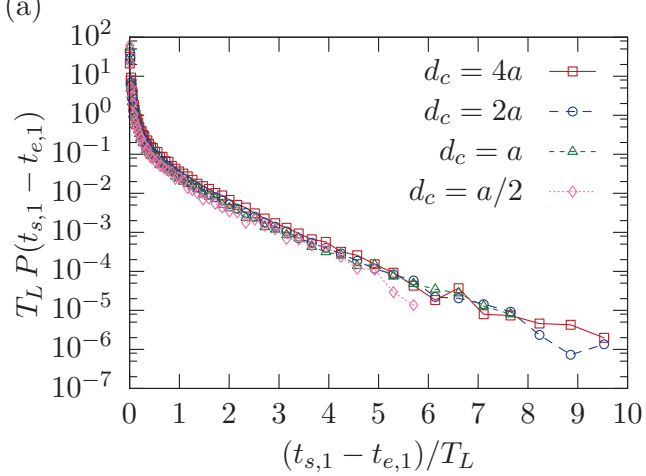

(b)

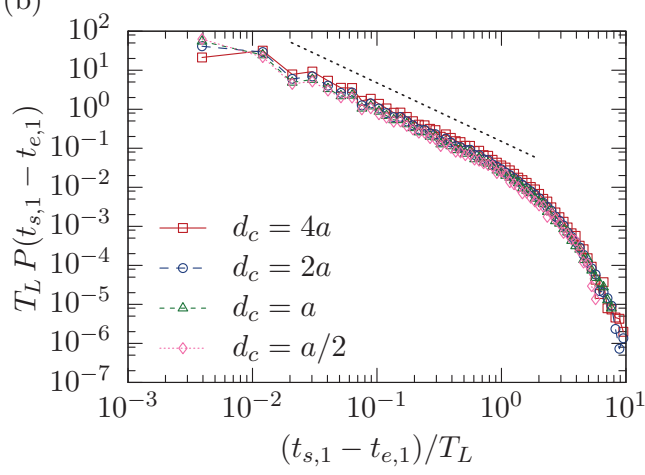

FIG. 5. (Color online) Lin-log (a) and $\log -\log (\mathrm{b})$ representations of the distribution of time spent together by pairs of particles during their first encounter. Several values of the critical distance $d_{c}$ are shown; the similarity between the curves shows that the dependence on $d_{c}$ is very weak (note that $d_{c} \ll \eta$ for all the values of $d_{c}$ shown). For comparison, the straight dotted line in (b) corresponds to a power law with exponent 1.5. The Stokes number used here is $\mathrm{St}=1.5$.

in units of the large eddy turnover time, $T_{L}$, the correlation time of the flow. Figure 5 shows an exponential decay of the PDF, with a characteristic time of the order of the large eddy turnover time. Thus, particles can spend together a time which is comparable to the large eddy turnover time (at least for the value of $\operatorname{Re}_{\lambda}$ used in our simulations). Figure 5(a) also indicates that the PDF has a very sharp maximum around $\Delta T_{1} \approx 0$. In fact, panel (b) shows the PDF in log-log units and suggests a power-law distribution of $\Delta T_{1}$. The exponent of the power law measured here is of the order -1.5 . As $\int_{\epsilon} x^{-1.5} d x$ diverges when $\epsilon \rightarrow 0$, the PDF necessarily saturates for time separations $\lesssim \Delta t$, where $\Delta t$ is the time at which we saved trajectories. Figure 5 shows the PDF of $\Delta T_{1}$, determined with several values of the critical distance $d_{c}$, very small compared to the Kolmogorov scale $\eta$ (we have $a / \eta \approx 1 / 12$ ). The PDFs are remarkably independent of $d_{c}$, at least provided the ratio $d_{c} / \eta$ is small, where $\eta$ is the Kolmogorov length.

Figure 6 shows the probability distribution function for $\Delta T_{n}$, with $n=2,3,4(n>1)$. The PDFs still exhibit for very long values of $\Delta T_{n}$ exponentially decaying tails [see panel (a)] with the same decay rate as obtained for $\Delta T_{1}$. However, the short time behavior of the PDF does not exhibit the very large peak seen in Fig. 5. In fact, the probability distribution does not exhibit any power law at short values of $\Delta T_{n}$, as seen in panel (b).

The difference between the statistical properties of $\Delta T_{1}$ and $\Delta T_{n}$ for $n \geqslant 2$ is therefore restricted to the short time behavior. In physical terms, the probability that the two particles do not spend much time together is much larger during the first encounter than during the following one. Particles can spend a short time when they are impacting each other with a large velocity difference. One may surmise that in such a case, particles will separate very fast and not get into close contact afterward. In other words, the events leading to several contacts are unlikely to have initially a small value of $\Delta T_{1}$. To actually check this, Fig. 6 also shows the PDF of $\Delta T_{1}$, conditioned on the fact that the two trajectories will come into contact more than one time (see the curve with the square symbols). As expected, conditioning the probability of $\Delta T_{1}$ on the fact that there are more than one encounter between the trajectories significantly reduces the probability for the particles of separating very fast. In this spirit, Fig. 7 shows the PDF of $\Delta T_{1}$ conditioned on having several successive encounters between the trajectories $(N>1)$ or simply one (a)

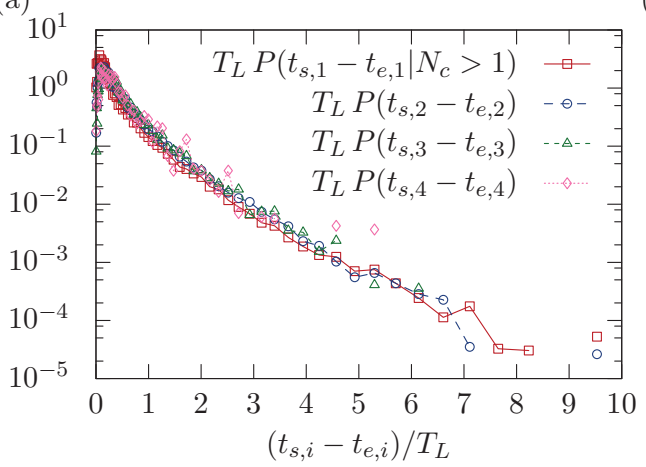

(b)

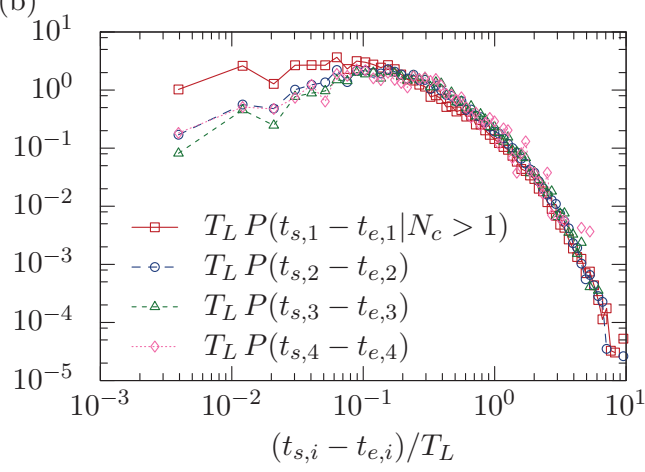

FIG. 6. (Color online) Lin-log (a) and log-log (b) representations of the distribution of time spent together by pairs of particles during second (circle), third (upward pointing triangle), and fourth (diamond) encounters. These distributions are remarkably similar. For comparison, the distribution of time that particles spend together during their first encounter, conditioned on the fact that they will meet again, is shown (square symbols). The cuspy distribution, observed for $t_{s, 1}-t_{e, 1}$, is not seen for these distributions. The Stokes number used here is St $=1.5$. 
(a)

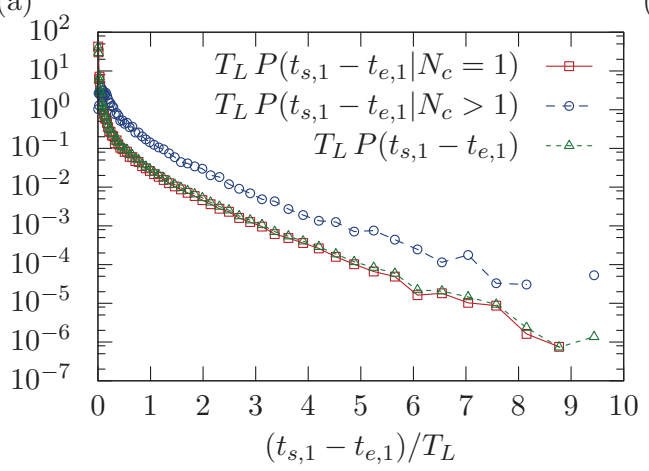

(b)

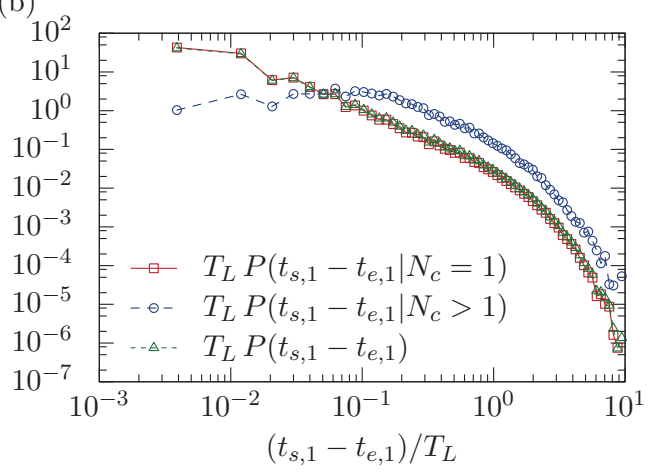

FIG. 7. (Color online) The distribution of the time spent together by two particles during their first encounter, $p\left(t_{s, 1}-t_{e, 1}\right)$ (upward pointing triangles), and the same distribution conditioned on the fact that trajectories will separate after their first encounter and not meet again $\left(N_{c}=1\right.$; square symbols). Finally the same PDF is shown again, this time conditioned on the fact that the particles will meet again $\left(N_{c} \geqslant 2\right.$; circles). The statistics is dominated by pairs that collide only once. The large probability, hence the power-law distribution, that two particles spend a short time together comes from trajectories with $N_{c}=1$. Both conditional probabilities show the exponential tail for large times. The Stokes number used here is $\mathrm{St}=1.5$.

$(N=1)$, together with the total PDF of $\Delta T_{1}$. As the probability of having multiple encounters between the two particles remains relatively small, the PDF of $\Delta T_{1}$ is extremely close to the PDF of $\Delta T_{1}$ conditioned on having $N=1$.

Aside from the difference between the PDF of $\Delta T_{1}$ and $\Delta T_{n}$ at short times, it appears that the process leading to subsequent encounters between particle trajectories is largely self-similar, i.e., does not depend much on the index $n>1$. This effect is strengthened by studying the difference between the time it takes for two trajectories to come into contact again. To this end, Fig. 8 shows the probability distribution function of $\Delta T_{n}^{e}=\left(t_{e, n+1}-t_{e, n}\right)$. The PDF is found to be remarkably similar (independent of $n$ ). As was the case for $\Delta T_{n}$, the PDF has an exponential tail at large values of $\Delta T_{n}^{e}$. The distribution, however, peaks at a finite value of $\Delta T_{n}^{e} \approx 0.6 T_{L}$.

\section{Stokes number dependence of the contact-time statistics}

The general picture, shown in Sec. IV A and IV B for particles with a Stokes number $\mathrm{St}=1.5$, has been found to be qualitatively unchanged when the Stokes number is varied.
However, the details differ quantitatively. This can be seen by representing the distribution of the time difference $\Delta T_{1}$ by an asymptotic fit of the form

$$
P\left(\Delta T_{1}\right) \approx \mathcal{N}\left(\Delta T_{1} / T_{L}\right)^{-\xi} \exp \left(-\kappa \Delta T_{1} / T_{L}\right) .
$$

The coefficient $\mathcal{N}$ in Eq. (16) is simply adjusted to enforce that the PDF is properly normalized. The coefficients $\xi$ and $\kappa$ are determined by fitting the PDFs. The quality of the fit is very good, as shown in Figs. 9(a) and 9(b), at least for $0.3 \lesssim$ St. The fitting parameters are found to depend very significantly on $\mathrm{St}$; see Fig. 10. In the very small Stokes number limit, the exponent $\xi$ diminishes, suggesting that the distribution $P\left(\Delta T_{1}\right)$ is becoming closer to a purely exponential distribution. In the opposite limit, the coefficient $\kappa$ decreases, whereas the power $\xi$ seems to increase.

\section{VELOCITY DIFFERENCE BETWEEN COLLIDING PARTICLES}

The time that two ghost particles spend in "contact" (that is, the length of time over which the separation of their centers
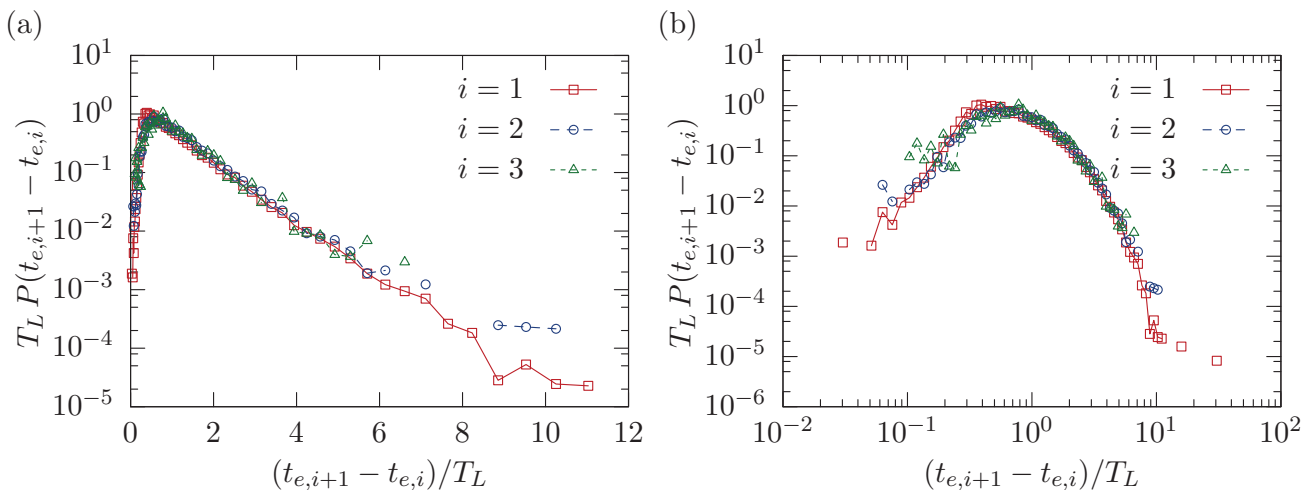

FIG. 8. (Color online) Statistics for successive encounters between two particle trajectories. Shown is the PDF of $t_{e, i+1}-t_{e, i}, t_{e, i}$ being defined in Fig. 4. (a) The data in lin-log scaling; (b) the same data in log-log scaling. The PDFs do not depend on $i$, suggesting a self-similar process. The Stokes number used here is $\mathrm{St}=1.5$. 
(a)

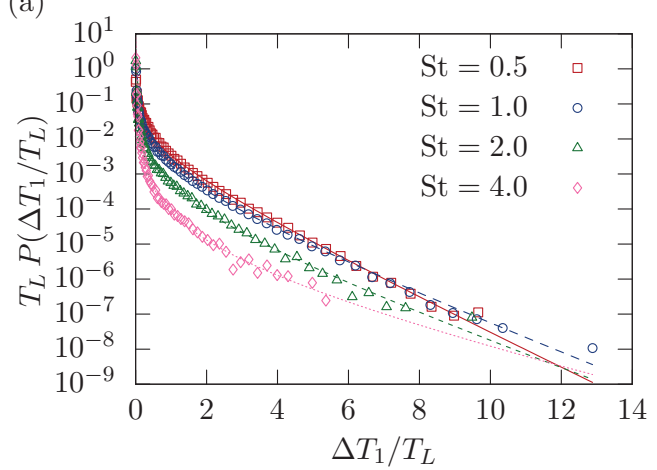

(b)

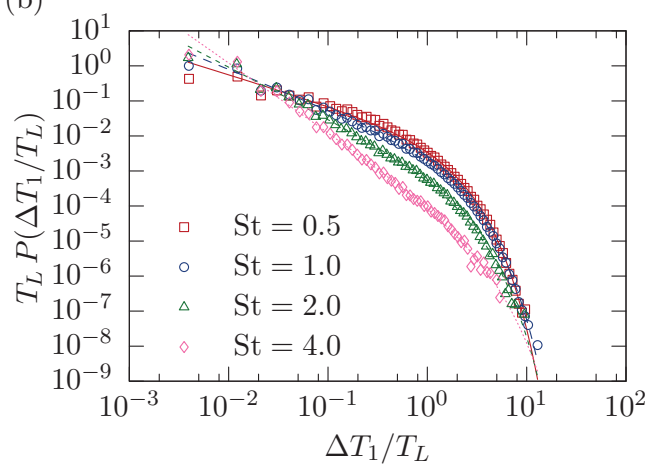

FIG. 9. (Color online) Fit of the PDF of $\Delta T$ by (16) at different values of the Stokes number in lin-log (a) as well as in log-log (b) scaling. The fits (lines) and the data (symbols) are shown for four values of the Stokes number, as indicated in the legend. The quality of the fit degrades at small values of St, suggesting that a more complicated functional form may be needed. The fit parameters are shown in Fig. 10 as a function of St.

satisfies $d \leqslant 2 a$ ) is determined by two factors: the impact parameter and the relative velocity of the collision. The data in Sec. IV show two striking aspects of the contact-time statistics. First, the statistics of the first contact are different from those of all the others. Second, there is evidence for a power-law regime in the distribution of the first contact time. In this section we discuss how both of these observations can be explained in terms of properties of the distribution of collision velocities.

\section{A. Multiple collisions happen at slow relative velocities}

The probability of the radial relative velocity, defined as $w_{r}=\delta \boldsymbol{v} \cdot \delta \boldsymbol{r} /|\delta \boldsymbol{r}|$, is shown in Fig. 11 for colliding particles and at $\mathrm{St}=1$. We remind that the PDF for colliding particles is different from the one for all particles in contact [35]. In panel (a) this PDF is shown for two different situations. In the first one, all colliding particles are taken into account, as is the case in the GCA. In the other one, only pairs that collide for the first time are taken into account. In both cases the bulk of the PDF is located at small values of $\left|w_{r}\right|$ and exhibits an exponential tail at very large collision velocities (see inset). A close comparison of the two probabilities shows that the contribution of small relative velocities corresponding to first collisions is smaller than for all particle pairs detected with the GCA. This suggests that the error in the GCA stems mainly from collisions with small relative velocities. The right panel

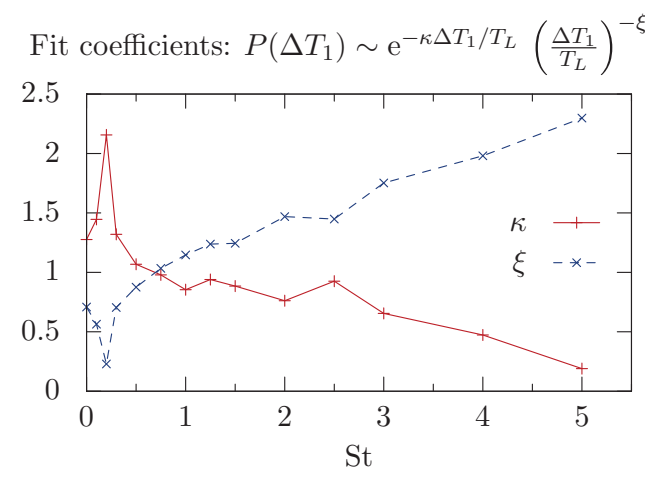

FIG. 10. (Color online) The PDF of $\Delta T$ has been fitted by Eq. (16) at different values of the Stokes number. The variation of the exponent $\xi$ and of the coefficient $\kappa$ are shown as a function of St. The quality of the fits can be examined in Fig. 9. of Fig. 11 extends this observation to different Stokes numbers by comparing the mean radial relative collision velocities obtained with both schemes. For Stokes numbers $\lesssim 2$ the collisions corresponding to particles in contact for the first time show on average slightly larger radial relative velocities. However, the averaged relative velocities for particle pairs colliding more than one time are significantly smaller than both the velocities of first collisions and the velocities in the GCA. This can be seen in the inset of the right panel. The observations summarized in Fig. 11 therefore demonstrate that multiple collisions occur at small relative velocity.

It has been argued that the collision rate between particles in a turbulent suspension can be resolved into two components, as represented by Eq. (2) [2,4]. In the decomposition (2), the term $\Gamma_{\text {adv }}$ represents collisions due to shearing motion, which occur with relative velocities of order $a / \tau_{\mathrm{K}}$, whereas the term $\Gamma_{\text {caust }}$ represents collisions between particles which are moving relative to the flow and on different branches of a phase-space manifold, separated by a caustic. The relative velocity of the collisions which contribute to $\Gamma_{\text {caust }}$ is much higher, of order $\left(\eta / \tau_{\mathrm{K}}\right) f(\mathrm{St})$, where $f(\mathrm{St})$ is an increasing function, which is of order unity at $\mathrm{St}=1$. The ideas underlying this decomposition also explain why the statistics of the first contact time are different from those of all the subsequent contacts. According to this picture, multiple collisions are almost exclusively due to the advective collision mechanism and are very unlikely for caustic-induced collisions because the high relative velocity rapidly moves the particles out of proximity. In the advective process multiple collisions arise because of temporal fluctuations of the shear rate tensor [9]. This model explains why the first collision has different contact time statistics, and why the multiple collisions have small relative velocities.

\section{B. Power-law distribution of contact times}

The evidence for a power law in the distribution of the contact time for the first collision is one of the conspicuous results of Sec. IV. In order to understand the origin of such power laws, we consider the following simplified model. We computed directly the distribution of the time $\Delta T$ that two particles in a gas of particles, with a Maxwellian distribution of velocity spent within a distance $2 a$ from each other. The 
(a)

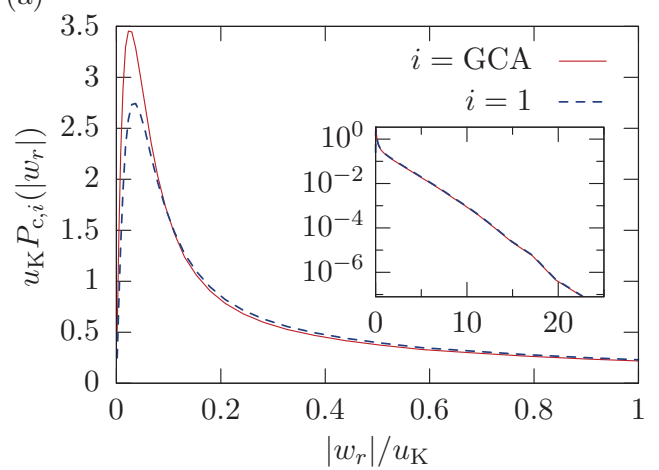

(b)

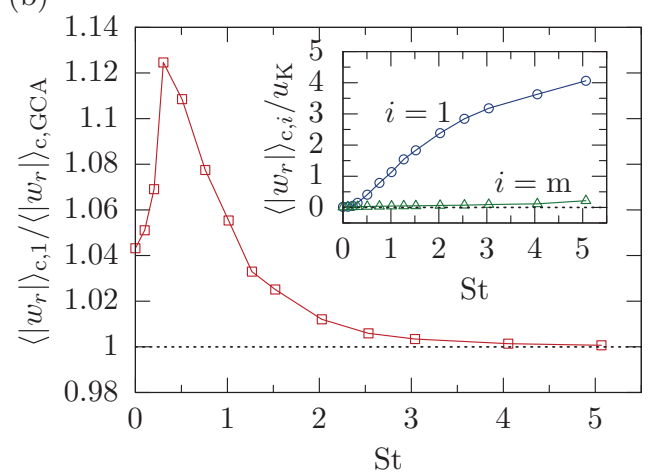

FIG. 11. (Color online) Probability of the radial relative collision velocity. (a) The full line corresponds to the PDF as obtained when taking into account all collisions, as in the GCA. The dashed line gives the PDF of solely first collisions. The figure has been obtained with a value of the Stokes number $\mathrm{St}=1.0$. The velocities are expressed in terms of the velocity $u_{\mathrm{K}}=\left(\eta_{\mathrm{K}} / \tau_{\mathrm{K}}\right)$ at the smallest scale. (b) The ratio of the two mean radial relative collision velocities $\left\langle\left|w_{r}\right|\right\rangle_{c, 1}$ and $\left\langle\left|w_{r}\right|\right\rangle_{c, \mathrm{GCA}}$ for different Stokes numbers. The former takes into account only first collisions; the latter incorporates all collisions detected within the framework of the GCA. Furthermore the inset in (b) shows $\left\langle\left|w_{r}\right|\right\rangle_{c, 1}$ and the radial relative velocity of multiple collisions $\left\langle\left|w_{r}\right|\right\rangle_{c, m}$ in terms of $u_{\mathrm{K}}$.

root mean square of one velocity component of these particles is taken to be $\sigma$. This model corresponds to the very large St limit of inertial particles in a turbulent flow. In that case the gas of particles reduces to particles each moving with its own velocity, distributed according to the Maxwell distribution [36]. A simple calculation leads to the following PDF of the time that two particles spend together:

$$
\begin{aligned}
P(\Delta T) & =2 \frac{\sigma}{a} \frac{1}{\zeta}\left(1-\exp \left(-\zeta^{2}\right)\left[1+\zeta^{2}+\frac{1}{2} \zeta^{4}\right]\right) \\
\text { with } \zeta & =\frac{2 a}{\sigma \Delta T} .
\end{aligned}
$$

In the limit of long times, $\Delta T \rightarrow \infty, \zeta \rightarrow 0$, the PDF in Eq. (17) reduces to

$$
P(\Delta T) \propto \frac{1}{\Delta T^{5}}
$$

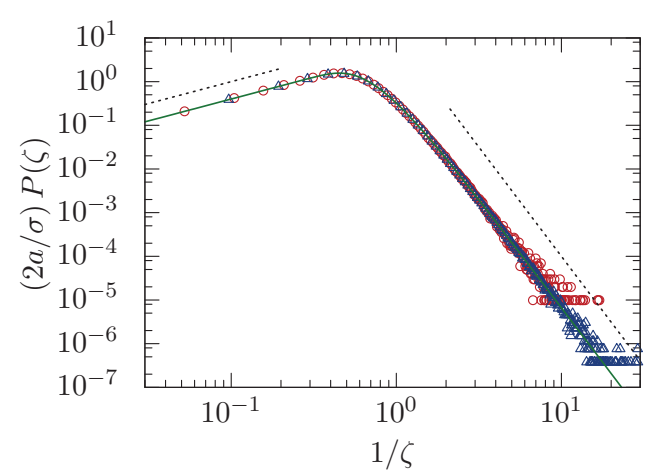

FIG. 12. (Color online) Comparison between the calculation of the time $\Delta T$ spent by two particles together, by simulating directly a gas of Maxwell particles (symbols) and the result of the analytic formula (17) (continuous line). The different symbols correspond to simulations with larger (circles) and smaller (triangles) particles. The time $\Delta T$ is expressed in terms of $\zeta$, defined by (18). The behavior of the distribution decays for large values of $\Delta T$ as $\sim \Delta T^{-5}$. This limit, as well as the short time behavior $\sim \Delta T$, are shown as dotted lines.
This result was checked directly by generating a gas of Maxwellian particles and determining the time particles spend together (Fig. 12). The distribution of $\Delta T$ is found in excellent agreement with (17). We furthermore note, that Eq. (17) displays another power law for short contact times. But this behavior is only apparent for $\Delta T<2 a / \sigma \ll \tau_{\mathrm{K}}$. In our DNS of the turbulent transport of particles we do not resolve these time scales.

Let us consider the implications of the model leading to Eq. (19) for the distribution of first contact times. The important point is to observe that a power law in the distribution of small relative velocities leads to a power-law distribution of long contact times. The data in Fig. 5 show a power-law distribution in the contact times at short times. We should, however, remember that the first contacts are a combination of caustic-mediated collisions (with high relative velocity) and advective collisions (with low relative velocity). We propose that the power-law distribution of the contact time results from the low-velocity tail of the caustic-mediated collisions having a PDF which can be approximated by a power-law at small velocities.

\section{CONCLUSION}

We have studied collisions in turbulent suspensions, with the aim of investigating the statistics of multiple collisions and of determining the systematic errors in the determination of the collision rate using the GCA for particles which aggregate upon contact.

We found that, after the first collision, multiple collisions involving the same pair of particles occur with a probability that decays exponentially with the number of collisions, $N_{c}$. We also studied the time that particle trajectories spend close to one another. We find the PDF of the time spent by two particles within a distance smaller than a critical value $d_{c}$ exhibits an exponential tail at very long times. At shorter times the contact time PDF of the first collision obeys a power law.

To study the errors induced by the GCA, we have compared the results using the GCA, and a more realistic algorithm, 
which consists in replacing one of the particles that underwent collision by a particle from a "reservoir." The error we find by using the GCA is as large as $\sim 15 \%$ at very small Stokes number and tends to decrease when St increases. These small, but systematic, errors should be taken into account when modeling the effect of turbulence in particle suspensions.

The present study rests on a numerical investigation at a relatively small Reynolds number $\left(\operatorname{Re}_{\lambda} \approx 130\right)$. We observed that the typical time scale over which the particles are in contact is comparable to the large eddy turnover time, $T_{L}$. However, a reasonable expectation is that the time that particles spend together is proportional to the inverse of the largest Lyapunov exponent, which has been measured to be of the order of $\sim 15 \tau_{\eta}$ and not of $\sim T_{L}$. The growth of the ratio $T_{L} / \tau_{\eta}$ with the Reynolds number $\left(T_{L} / \tau_{\eta} \propto \operatorname{Re}_{\lambda}\right)$ suggests that at higher $\operatorname{Re}_{\lambda}$, the fluid motion should become more persistent over a time scale $\sim 15 \tau_{\eta}$. For this reason we do not expect that the effects of multiple collisions decrease when $\operatorname{Re}_{\lambda}$ increases.

\section{ACKNOWLEDGMENTS}

We thank Bernhard Mehlig and Kristian Gustavsson for discussions, and Matthäus Bäbler for pointing out Ref. [21]. A.P. and E.L. acknowledge the financial support of ANR (contract TEC 2). The numerical calculations have been performed at the PSMN computer center of the Ecole Normale Supérieure in Lyon. M.W. and A.P. were supported by the EU COST action MP0806 "Particles in Turbulence."

\section{APPENDIX}

In this Appendix we consider the equivalence of two different approaches to determining the true rate of first contact collisions, $\Gamma_{1}$, and therefore provide evidence that $\Gamma_{1}$ is indeed the physically appropriate definition of the collision rate, for a class of systems where particles react only at first contact. One approach is to use ghost particles and count the rate of multiple collisions, so that $\Gamma_{1}=\Gamma_{\mathrm{GCA}}-\Gamma_{m}$. The alternative approach is to use one of the two "substitution schemes," described in Sec. II C, leading to rates $\Gamma_{\mathrm{Re} 1}$ and $\Gamma_{\mathrm{Re} 2}$. In this
Appendix we show that these estimates differ as a result of finite particle density effects. We provide evidence, however, that they become equal in the limit of very dilute systems (that is, as the particle density approaches zero).

Figure 13 shows the collision rates produced by these algorithms, as well as $\Gamma_{1}$, obtained at several values of the volume fraction $\Phi: \Phi_{0}, \Phi_{0} / 2$, and $\Phi_{0} / 4$. The numerical results show that

(1) $\Gamma_{\mathrm{Re} 2}(\Phi)<\Gamma_{\operatorname{Re} 1}(\Phi)<\Gamma_{1}=\Gamma_{\mathrm{GCA}}-\Gamma_{m}$,

(2) $\Gamma_{\operatorname{Re} 2}(\Phi / 2)=\Gamma_{\operatorname{Re} 1}(\Phi)$.

A more careful analysis of the data reveals that when $\Phi \rightarrow$ 0 , both $\Gamma_{\operatorname{Re} 1}$ and $\Gamma_{\mathrm{Re} 2}$ tend to $\Gamma_{\mathrm{GCA}}-\Gamma_{m}$, with corrections which are linear in $\Phi$. Thus, our numerical results demonstrate that the results from the three different algorithms agree in the dilute limit, $\Phi \rightarrow 0$.

The numerical results can be understood in the following way. We introduce the coefficients $R=\Gamma n$, which determine the rate of collision of a test particle moving in a medium containing other particles with number density $n$. The ergodic assumption implies that the actual collision rate can be inferred from the long-time behavior of a single test trajectory.

The quantity $n \Gamma_{\mathrm{GCA}}$ is just the total rate of collision along the test trajectory, and $n \Gamma_{m}$ is the rate for collisions in which the test particle encounters the same target particle more than once. For systems where there is coalescence on contact, only the rate of first collision is of interest. The preferential concentration effect enhances the rate of subsequent collisions with further particles, once a pair of particles has collided. However, in the limit $n \rightarrow 0$, the time between collision events approaches infinity $(\propto 1 / n)$. For this reason, in the limit as $n \rightarrow 0$ the first contact collision rate is precisely $n \Gamma_{\text {Rei }}$.

In terms of the long-time average over trajectories, the algorithm which yields $\Gamma_{\mathrm{Re} 2}$ changes the position of the trajectory, therefore destroying the possibility of further collisions with the surrounding environment. In addition, a different realization of the surrounding background particles is chosen. In contrast, for the algorithm which yields $\Gamma_{\mathrm{Re} 1}$, either the position of the test trajectory, or the background of surrounding particles, is changed, each with a probability $1 / 2$. (a)

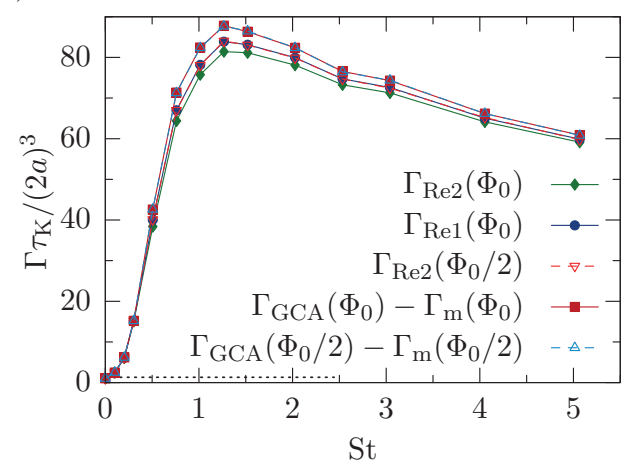

(b)

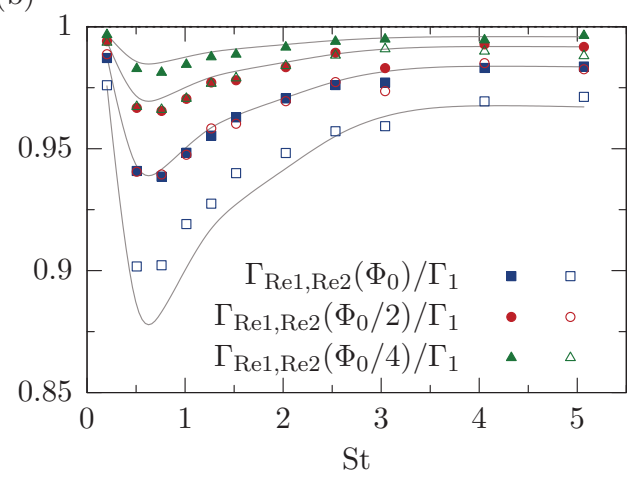

FIG. 13. (Color online) Comparison between $\Gamma_{1}=\Gamma_{\mathrm{GCA}}-\Gamma_{m}$, and the collision rates obtained by using the substitution algorithms ( $\Gamma_{\mathrm{Re} 1}$ and $\left.\Gamma_{\mathrm{Re} 2}\right)$. The left panel shows the raw data for some of the different collision kernels as indicated in the legend. The right panel shows $\Gamma_{\mathrm{Re} i} / \Gamma_{1}$ as a function of the Stokes number for those runs listed in Table I for which $\Phi_{0}=4.5 \times 10^{-5}$. The open (filled) symbols correspond to the algorithm $\operatorname{Re} 2(\operatorname{Re} 1)$. The full lines are deduced from each other by multiplication by a factor 2 and suggest that the dependence of the difference $\Gamma_{1}-\Gamma_{\text {Rei }}$ behaves linearly with the density of particles in the system. 
Changing the position of the test particles or the surrounding background results, first, in preventing any further collision between the pair of particles that came into contact. The simplest approximation for the probability per unit time of a collision between the test particle and a third particle, after a contact has been detected, is proportional to $R$. This suggests that the approximations $\Gamma_{\mathrm{Re} 1}$ or $\Gamma_{\mathrm{Re} 2}$ miss a correction of order $O(R \tau)$, where $\tau$ is a characteristic time over which the surrounding particles rearrange. Moreover, the algorithm yielding $\Gamma_{\mathrm{Re} 2}$ misses twice as many collisions with a third particle as $\Gamma_{\mathrm{Re} 1}$, which is also consistent with our numerical observations. These arguments suggest that

$$
\Gamma_{\mathrm{Re} i}=\Gamma_{1}[1-O(i R \tau)] .
$$

As $R \propto n$, this expression justifies the numerical observation that $\Gamma_{\operatorname{Re} 1,2}$ are smaller than $\Gamma_{1}$ and differ from it by a quantity proportional to $n$.
[1] P. G. Saffman and J. S. Turner, J. Fluid Mech. 1, 16 (1956).

[2] G. Falkovich, A. Fouxon, and M. G. Stepanov, Nature (London) 419, 151 (2002).

[3] R. A. Shaw, Annu. Rev. Fluid Mech. 35, 183 (2003).

[4] M. Wilkinson, B. Mehlig, and V. Bezuglyy, Phys. Rev. Lett. 97, 048501 (2006).

[5] V. S. Safranov, Evolution of Protoplanetary Cloud and Formation of Earth and Planets, Israel Program for Scientific Translations (Keter Publishing House, Jerusalem, 1972).

[6] M. Wilkinson, B. Mehlig, and V. Uski, Astrophys. J. Suppl. 176, 484 (2008).

[7] L.-P. Wang, A. S. Wexler, and Y. Zhou, Phys. Fluids 10, 266 (1998).

[8] J. Chun and D. L. Koch, Phys. Fluids 17, 027102 (2005).

[9] K. Gustavsson, B. Mehlig, and M. Wilkinson, New J. Phys. 10, 075014 (2008).

[10] G. Krstulovic, M. Cencini, and J. Bec, J. Stat. Phys. 153, 530 (2013).

[11] M. C. Jullien, J. Paret, and P. Tabeling, Phys. Rev. Lett. 82, 2872 (1999).

[12] M. P. Rast and J. F. Pinton, Phys. Rev. Lett. 107, 214501 (2011).

[13] R. Scatamacchia, L. Biferale, and F. Toschi, Phys. Rev. Lett. 109, 144501 (2012).

[14] G. Falkovich and A. Pumir, J. Atmos. Sci. 64, 4497 (2007).

[15] G. P. Bewley, E.-W. Saw, and E. Bodenschatz, New J. Phys. 15 083051 (2013).

[16] K. Gustavsson and B. Mehlig, Phys. Rev. E 84, 045304(R) (2011).

[17] K. Gustavsson and B. Mehlig, arXiv:1309.3834[physics. flu-dyn].

[18] M. Voßkuhle, A. Pumir, E. Lévêque, and M. Wilkinson, arXiv:1307.6853[physics.flu-dyn].
[19] J. Bec, S. Musacchio, and S. S. Ray, Phys. Rev. E 87, 063013 (2013).

[20] M. Voßkuhle, A. Pumir, and E. Lévêque, J. Phys.: Conf. Ser. 318, 052024 (2011).

[21] J. B. Jørgensen, J. Mann, S. Ott, H. L. Pécseli, and J. Trulsen, Phys. Fluids 17, 035111 (2005).

[22] A. J. Vidgop and I. Fouxon, Phys. Rev. E 84, 055202 (2011).

[23] U. Frisch, Turbulence: The Legacy of A. N. Kolmogorov (Cambridge University Press, Cambridge, 1995).

[24] A. G. Lamorgese, D. A. Caughey, and S. B. Pope, Phys. Fluids 17, 05106 (2005).

[25] E. Calzavarini, R. Volk, M. Bourgoin, E. Lévêque, J.-F. Pinton, and F. Toschi, J. Fluid Mech. 630, 179 (2009).

[26] S. A. Orszag, J. Atmos. Sci. 28, 1074 (1971).

[27] M. R. Maxey and J. J. Riley, Phys. Fluids 26, 883 (1983).

[28] R. Gatignol, J. Mec. Theor. Appl. 2, 143 (1983).

[29] W. H. Press, S. A. Teukolsky, W. T. Vetterling, and B. P. Flannery, Numerical Recipes: The Art of Scientific Computing, 3rd ed. (Cambridge University Press, Cambridge, 2007).

[30] S. Sundaram and L. R. Collins, J. Comput. Phys. 124, 337 (1996).

[31] Y. Zhou, A. S. Wexler, and L.-P. Wang, Phys. Fluids 10, 1206 (1998).

[32] S. Sundaram and L. R. Collins, J. Fluid Mech. 335, 75 (1997).

[33] B. Rosa, H. Parishani, O. Ayala, W. W. Grabowski, and L.-P. Wang, New J. Phys. 15, 045032 (2013).

[34] J. Bec, L. Biferale, G. Boffetta, M. Cencini, S. Musacchio, and F. Toschi, Phys. Fluids 18, 091702 (2006).

[35] L.-P. Wang, A. S. Wexler, and Y. Zhou, J. Fluid Mech. 415, 117 (2000).

[36] J. Abrahamson, Chem. Eng. Sci. 30, 1371 (1975). 